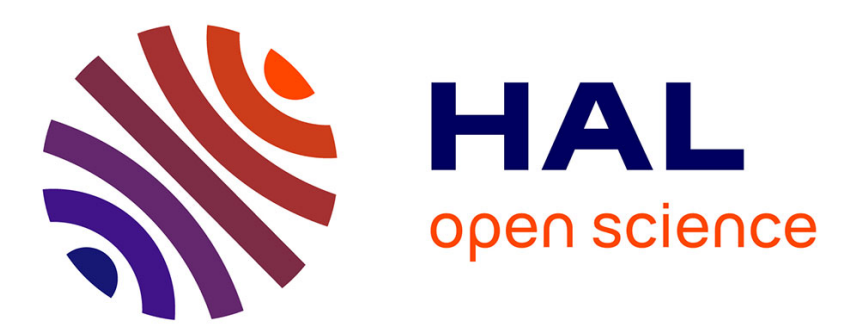

\title{
Connaître la gymnastique et savoir l'enseigner en EP : la nature de la connaissance pédagogique des contenus et son lien à l'instruction en classe
}

Marc Cizeron, Nathalie Gal-Petitfaux

\section{- To cite this version:}

Marc Cizeron, Nathalie Gal-Petitfaux. Connaître la gymnastique et savoir l'enseigner en EP : la nature de la connaissance pédagogique des contenus et son lien à l'instruction en classe. Science et Motricité : revue scientifique de l'ACAPS / ACAPS, 2005, 55 (2), pp.9-33. hal-01076167

\section{HAL Id: hal-01076167 \\ https://hal.science/hal-01076167}

Submitted on 21 Oct 2014

HAL is a multi-disciplinary open access archive for the deposit and dissemination of scientific research documents, whether they are published or not. The documents may come from teaching and research institutions in France or abroad, or from public or private research centers.
L'archive ouverte pluridisciplinaire HAL, est destinée au dépôt et à la diffusion de documents scientifiques de niveau recherche, publiés ou non, émanant des établissements d'enseignement et de recherche français ou étrangers, des laboratoires publics ou privés. 
Cizeron, M., Gal-Petitfaux, N. (2005). Connaître la gymnastique et savoir l'enseigner en EP : la nature de la connaissance pédagogique des contenus et son lien à l'instruction en classe, Science et Motricité, 55(2), 9-33.

\section{Résumé}

Le but de l'étude était d'analyser, dans une perspective située, la connaissance pédagogique de la gymnastique sur laquelle s'appuient les enseignants d'EP expérimentés pour guider les apprentissages des élèves. Les participants à l'étude étaient des enseignants spécialistes de gymnastique, expérimentés dans l'enseignement scolaire de cette activité. Des données d'observation des communications des enseignants en classe et d'entretiens post-leçons ont été recueillies. Les premières ont été traitées en distinguant les aspects locutoires et illocutoires des communications verbales, et en leur appliquant une analyse de contenu. Les secondes ont été soumises à une analyse de discours portant sur les arguments mobilisés par les enseignants. Les résultats montrent que cette connaissance est structurée par des formes typiques du corps et des mouvements gymniques des élèves, que les enseignants reconnaissent en classe et interprètent immédiatement en termes d'efficacité : alors que les élèves sont en activité motrice, les enseignants perçoivent les formes de leur corps et de leurs mouvements en leur attribuant directement une signification et en sachant immédiatement comment intervenir. Cette reconnaissance perceptive, en contexte, permet de comprendre comment leur connaissance pédagogique de la gymnastique est couplée à leur activité d'instruction en classe : elle a une valeur fonctionnelle comme disposition à agir.

Titre courant : Connaissance pédagogique de la gymnastique

Mots clefs : Activité / Connaissance pédagogique des contenus / Education Physique / Gymnastique / Instruction 
Title : Knowledge of gymnastics and knowing how to teach it to PE pupils: the nature of the pedagogical knowledge content and its relationship with classroom instruction

\begin{abstract}
The aim of this study was to analyze, in a situated perspective, pedagogical knowledge of gymnastics which is used by accomplished PE teachers to guide pupil learning. The classroom teacher's communications and post-lesson interviews were collected as data. The results show that teachers knowledge is structured by the pupils' typical body shapes and gymnastics movements. While the pupils are moving, the teachers perceive and directly attribute meanings to body shapes and movements. Thus, they immediately know how to act. This perceptive recognition in context shows that pedagogical knowledge of gymnastics is coupled with instructional activity in classroom: it has a functional value in terms of predisposition for acting.
\end{abstract}

Running-title : Pedagogical Knowledge in gymnastics

Key-words : Activity / Pedagogical Content Knowledge / Physical education / Gymnastics / Instruction 


\section{Introduction}

\subsection{Les connaissances et l'activité en classe des enseignants}

Dans le domaine des recherches sur l'enseignement, de nombreux travaux se sont intéressés aux connaissances de l'enseignant. C'est sous l'expression de «base de connaissances », issue de l'approche cognitiviste, que sont désignés de façon globale tous les savoirs de l'enseignant.

Les recherches ont révélé une diversité de connaissances à la base du travail de l'enseignant. Malgré leur éclatement dû aux perspectives théoriques variées et aux objets multiples (savoirs, connaissances, habiletés, compétences, savoir-faire) investis par les chercheurs, ces résultats ont néanmoins conduit à l'identification de champs homogènes de connaissance (Leinhardt \& Greeno, 1986 ; Shulman, 1986). Shulman (1987) a notamment distingué sept catégories de connaissances propres aux enseignants. Trois d'entre elles sont liées aux contenus : la connaissance de la matière, la connaissance pédagogique des contenus (Pedagogical Content Knowledge ou PCK)1, la connaissance des programmes ; et quatre autres concernent la pédagogie générale, les caractéristiques des élèves, le contexte éducatif, et les objectifs de l'éducation. Les PCK sont apparus d'emblée comme un domaine primordial et spécifique : la connaissance des contenus de la matière réellement utilisée en classe par les enseignants pour instruire les élèves.

Considérées comme un mélange de connaissance de la matière et de connaissance pédagogique (Shulman, 1986), ou comme connaissances pédagogiques spécifiques d'une matière (Tamir, 1988), les PCK ont été l'objet de nombreuses recherches en enseignement (Gess-Newsome \& Lederman, 1999, pour une revue). La façon dont elles sont aujourd'hui définies et étudiées repose sur deux préoccupations centrales des chercheurs : d'une part, caractériser la nature du contenu des PCK ; d'autre part, expliquer la nature de leur relation à la pratique de l'enseignant.

Partant de la définition de Shulman (1986), les PCK ont d'abord été caractérisées comme catégorie spécifique et séparée de connaissance. Puis, s'intéressant de plus près à la façon dont elles interagissaient avec les valeurs de l'enseignant (Gudmundsdottir, 1990), avec leurs stratégies d'instruction, les media utilisés en classe, les objectifs éducatifs (Grossman, 1990 ; Marks, 1990), les chercheurs les ont progressivement définies comme l'intégration de quatre catégories de connaissance en interaction permanente : la connaissance de la matière, des élèves, du contexte scolaire, et de la pédagogie (Grossman, 1990 ; Marks, 1990). Ces catégories sont liées entre elles par des relations dynamiques, au point de devenir inséparables les unes des autres : elles se transforment et s'intègrent perpétuellement selon un processus continu de connaissance de la matière dans le contexte de la classe (Pedagogical Content Knowing) (Cochran, De Ruiter, \& King, 1993).

\footnotetext{
${ }^{1}$ Amade-Escot (1998) signale que la traduction usuelle de l'expression « Pedagogical Content Knowledge » est « connaissance des contenus pédagogiques ». Elle estime toutefois que cette traduction est peu satisfaisante, et lui préfère la locution de « connaissances didactiques » qui, selon elle, rendrait compte plus finement du registre de connaissances explorées par les recherches qui utilisent l'expression de PCK. Nous préférons la traduction « connaissance pédagogique des contenus » car elle met l'accent sur le caractère pédagogique de la connaissance de la matière enseignée, conformément à la définition que Shulman en donnait initialement. C'est donc cette dernière expression qui sera utilisée comme traduction de l'expression PCK dans cet article.
} 
Si les recherches ont montré l'existence de relations entre les connaissances des enseignants et leurs pratiques en classe, la nature exacte de ces relations reste peu investiguée (Baxter \& Lederman, 1999). Les études montrent toutefois que ces connaissances ne sont pas isolables du contexte pratique de leur usage : elles ne sont pas déposées en mémoire, sous la forme d'une base de connaissance consciemment identifiée par les enseignants, puis simplement appliquée en contexte pratique. Elles sont tacites et fortement enchâssées, encapsulées (Durand, 1996), dans les actions (Shulman, 1987 ; Tochon, 1991) ; elles s'actualisent dans des savoir-faire (Durand, 1996); elles sont ouvragées, au sens où elles caractérisent le « savoir au travail » (Tardif \& Lessard, 1999), et sont en relation étroite avec les conditions concrètes de la classe (Gauthier, Desbiens, Malo, Martineau, \& Simard, 1997).

\subsection{La connaissance pédagogique des contenus en Education Physique}

Les études menées sur les PCK dans le domaine de l'enseignement de l'Education Physique (PCK PE) ont confirmé le caractère contextuel de ces connaissances et leur imbrication à l'action de l'enseignant en classe. Elles sont décrites comme procédurales (Siedentop \& Eldar, 1989), expérientielles (Dodds, 1995 ; Rovegno, 1992 ; Sebren, 1995), enchâssées dans les pratiques d'enseignement (Rovegno, 1994 ; Sebren, 1995) ; dépendantes de contraintes contextuelles (Graber, 1995 ; Rovegno, 1994); et encapsulées dans les actions pratiques (Rovegno, 1992). Le caractère fortement contextualisé des PCK PE souligne combien l'expérience des enseignants est importante dans leur développement (Graber, 1995 ; Rovegno, 1992). Les enseignants débutants notamment, mobilisent peu de PCK PE dans le contexte de leurs leçons (Graber, 1995 ; Kulinna, Silverman, \& Keating, 2000). Même lorsqu'ils ont une bonne connaissance de la matière, ils ne savent pas comment ils doivent la connaître pour pouvoir l'enseigner à des élèves. Ces résultats sont confirmés par les recherches en didactique de l'EP. Amade Escot (1998, p. 224) indique que les contenus d'enseignement « relèvent d'une analyse de l'action contextualisée ».

Ces difficultés repérées chez les enseignants débutants s'expliquent par le fait que les PCK PE ne sont pas tant des connaissances préalables que les enseignants auraient à intégrer à leur pratique, que des connaissances qu'ils découvrent et construisent progressivement, pendant qu'ils agissent en classe, en mettant en relation la matière, les élèves, le contexte, et leurs intentions éducatives. Les PCK PE émergent du couplage action-situation et peuvent être qualifiées de connaissances situées (Rovegno, 1992). Rovegno (1992) insiste sur le lien organique qui unit l'action et la connaissance, et notamment sur l'importance de l'activité perceptive de l'enseignant dans cette relation.

Dans leur étude sur les PCK PE, Jenkins \& Lou Veal (2002) se centrent sur : a) les composantes du management de la classe (règles et routines pour l'installation du matériel, la façon de donner les instructions pour que les élèves écoutent) ; b) la connaissances des élèves (e.g., leurs stades de développement, leurs stratégies d'apprentissage, leurs connaissances préalables et leurs attitudes face à la matière enseignée) ; c) les démarches d'instruction (e.g., progression et adaptation des tâches aux élèves, stratégies d'instruction directe ou indirecte, approche centrée sur l'enseignant ou centrée sur l'élève, importance du langage et du vocabulaire utilisé dans la leçon) ; d) le contexte de la leçon (e.g., contexte spatial et temporel de la leçon, le matériel pédagogique). Cette dispersion des objets de recherche est devenue si forte que l'expression PCK PE est utilisée pour désigner de façon générique les connaissances 
professionnelles des enseignants (Amade-Escot, 1998), chaque chercheur ayant développé la définition initiale de Shulman en fonction de ses propres orientations (Gess-Newsome, 1999).

Une façon d'éviter cette dispersion est de recentrer l'objet des PCK PE sur leur définition initiale, c'est-à-dire un mélange de connaissance de la matière à enseigner et de connaissance pédagogique (Shulman, 1986). En d'autres termes, il s'agit de focaliser l'étude des PCK PE sur leur référence à la matière d'enseignement. Cependant, une difficulté est à surmonter : la constitution historique de l'EP comme discipline d'enseignement a conduit à rendre difficile l'identification de la matière à la base de son enseignement (Léziart, 1997 ; Siedentop, 2002). Les savoirs de référence (Léziart, 1997) ou les connaissances qui composent le champ de la matière (Siedentop, 2002), à partir desquels s'édifie l'enseignement de l'EP peuvent intégrer de nombreuses composantes. Les auteurs distinguent : les capacités et habiletés motrices ; les performances sportives, les habiletés sportives propres aux activités physiques et sportives ; les aspects mécaniques, physiologiques ou cognitifs liés à la pratique de ces habiletés sportives ; les connaissances historiques et culturelles sur les activités physiques et sportives ; les manières d'être, de faire, de sentir, de se penser, de se mouvoir, d'être avec les autres; les états émotionnels ; les normes, valeurs et traditions du sport ; les réponses comportementales. Dans cette étude, les techniques sportives propres aux activités sportives ont été retenues comme dimension de la matière de l'EP.

\subsection{Perspectives situées pour l'analyse des PCK PE}

Les études portant précisément l'attention sur la connaissance qu'ont les enseignants de la matière font défaut en éducation physique (Graber, 2001). Bien que les recherches sur les PCK PE s'intéressent à la connaissance intégrée de la matière que possèdent les enseignants, elles se sont centrées sur les caractéristiques générales de ces connaissances (Rovegno, Chen, \& Todorovich, 2003). Seules quelques rares études ont analysé les PCK d'enseignants d'EP confirmés en s'intéressant précisément à leurs contenus spécifiques dans une activité sportive particulière : les PCK relatives à l'enseignement du « jeu de crosse $»^{2}$ (Barrett \& Collie, 1996), et du dribble en Basket-ball (Rovegno et al, 2003).

Pour l'enseignement de la gymnastique, les recherches ont plutôt analysé les connaissances propres à son enseignement au travers des publications didactiques de référence (Nouillot et Nata, 2002 ; Robin, 1998), des discours des leaders d'opinions (Robin, 1998), et des pratiques usuelles déclarées (Carnus, 2002). L'étude ici vise plus particulièrement à analyser la connaissance de la matière qui structure les actes d'instruction en classe des enseignants, notamment lorsqu'ils guident les élèves pour leur faire apprendre les habiletés motrices gymniques. Etant donné le lien que les PCK PE entretiennent avec la connaissance de la matière et avec l'expérience de la classe, nous avons choisi de nous intéresser à des enseignants d'EP spécialistes de cette activité sportive, et expérimentés pour l'enseigner.

Le cadre théorique de la recherche s'inscrit dans le paradigme de l'action située, développé dans une perspective anthropo-phénoménologique (Cizeron \& Gal-Petitfaux, 2002, 2003 ; Gal-Petitfaux \& Durand, 2001 ; Malet, 2000). La connaissance et l'action sont

\footnotetext{
${ }^{2}$ Nous avons traduit par « jeu de crosse » le sport nommé « lacrosse », mentionné dans l'article de Barrett \& Collie (1996).
} 
envisagées comme structurellement couplées (Varela, 1989), et co-définies. Ceci revient notamment à mettre radicalement en suspens l'idée que les PCK PE sont des connaissances qui existent en dehors du contexte de l'activité des enseignants en classe. Le point de départ de l'étude a donc consisté à observer les actions de l'enseignant en situation de classe, pour identifier la connaissance de la matière à laquelle il se référait pour instruire. Les observations ont porté sur les communications verbales de l'enseignant, à partir desquelles ont été inférées leurs PCK PE.

Le choix d'étudier les communications verbales repose sur deux arguments : d'une part, les communications représentent une dimension du savoir-faire que les enseignants mettent en œuvre pour guider les apprentissages ; d'autre part, le suivi des paroles en situation permet « l'accès aux implicites qui s'y projettent en creux et éclaire toute une part de l'arrière-plan des interactions »(Grosjean \& Lacoste, 1999, p. 12).

\section{Méthode}

\subsection{Participants}

Huit enseignants d'EP spécialistes de gymnastique (quatre femmes et quatre hommes, âgés de 31 à 45 ans), ont été volontaires pour participer à cette étude. Ils avaient une expérience de plusieurs cycles annuels de gymnastique en EP et de l'encadrement hebdomadaire de la gymnastique à $1^{\prime} \mathrm{AS}^{3}$. Ils étaient tous : a) diplômés d'état en gymnastique sportive ; b) des anciens gymnastes ayant un vécu compétitif. Ils exerçaient dans différents collèges avec des classes mixtes, composées d'élèves âgés entre onze et quinze ans ne posant pas de problèmes particuliers, notamment de discipline.

\subsection{Recueil de données}

Chaque enseignant a été filmé au cours d'une leçon entière, saisie au milieu d'un cycle dont la durée était comprise entre huit et douze leçons. Les verbalisations émises ont été enregistrées à l'aide d'un caméscope équipé d'un micro HF.

La méthodologie adoptée, inspirée de l'ethnométhodologie (Coulon, 1988 ; Mehan \& Wood, 1975), a consisté à recueillir deux types de données : i) des enregistrements des communications verbales de l'enseignant en classe destinées à guider les élèves dans l'apprentissage des habiletés gymniques ; ii) des verbalisations de l'enseignant recueillies au cours d'entretiens post-leçons. Pour ces dernières, la technique de rétroaction vidéo a été utilisée, dans le cadre théorique de la co-construction sociale et culturelle des connaissances dans la réflexion partagée (Tochon, 1996, pour une revue). La rétroaction vidéo sert ici à provoquer des verbalisations concomitantes de l'analyse d'un enregistrement vidéo. Elle aide l'enseignant et le chercheur à explorer en commun les connaissances implicites qui guident l'action (Tochon, 1996).

\footnotetext{
${ }^{3}$ L'AS est l'Association sportive de l'établissement scolaire : les élèves inscrits y pratiquent une ou plusieurs activités sportives facultatives, en prolongement de l'EP obligatoire, et participent aux compétitions organisées par l'Union nationale du sport scolaire (UNSS).
} 
L'enseignant était invité, grâce à la situation de rétroaction vidéo, à se focaliser sur ses communications en classe pour les décrire et les argumenter à deux niveaux : ce qu'il cherchait précisément à obtenir comme transformation ou comme comportement chez les élèves; et ce qui l'avait incité à communiquer compte tenu de ce qu'il avait vu.

\subsection{Traitement des données}

L'analyse des données de communication de l'enseignant en classe a été réalisée à deux niveaux, selon les catégories d'actes de langage de Austin (1970) : a) les actes de locution, correspondant à ce que l'enseignant énonçait pour renseigner les élèves sur ce qu'ils réalisaient, ou sur ce qu'ils devaient faire ou ne pas faire pour acquérir les habiletés gymniques ; b) les actes d'illocution, par lesquels les enseignants rendent manifestes leurs intentions envers les élèves.

Pour les actes de locution, les données ont été catégorisées selon une analyse de contenu utilisant un modèle mixte (L'Ecuyer, 1988), dans lequel une partie des catégories étaient prédéterminées (modèle fermé), d'autres catégories apparaissant en cours d'analyse (modèle ouvert). L'analyse a d'abord porté sur un dénombrement exhaustif des termes adressés aux élèves par l'enseignant. Ces termes ont ensuite été répertoriés selon des catégories émergeantes. Par exemple, les termes pousser ou tomber ont été rapportés à la catégorie cinématique de description des mouvements, tandis que les termes dur ou relâché ont été classés dans la catégorie dynamique. Pour retranscrire les actes d'illocution des enseignants en classe, la ponctuation a été utilisée afin de rendre compte de la longueur et de la tonalité des phrases énoncées, en distinguant les phrases exclamatives ${ }^{4}$ des phrases indicatives ${ }^{5}$. Par exemple, le contenu de l'énoncé oral écouté sur les enregistrements audio-visuels «bras aux oreilles repoussez vous et grandissez vous au maximum » a été reporté en tenant compte de la façon dont l'enseignant l'avait exprimé, en l'occurrence sous la forme de deux phrases exclamatives : «bras aux oreilles! repoussez vous et grandissez vous au maximum! ». Un double codage a été réalisé pour garantir la fiabilité du report de la ponctuation. Un accord inter-codeurs de $91 \%$ a été obtenu après entraînement. Les textes verbatim des communications verbales des enseignants ont été soumis à une analyse statistique à l'aide d'un logiciel d'analyse statistique et sémantique (Cordial pro). Les analyses grammaticale et stylistique de ce logiciel ont fourni des informations quantitatives et qualitatives sur la forme des énoncés transcrits.

Les données recueillies au cours des entretiens ont permis d'affiner et compléter la documentation première sur les communications en classe. Elles ont été traitées selon deux volets : a) une analyse de contenu utilisant le modèle mixte de L'Ecuyer (1988) ; b) une analyse du discours d'explicitation portant sur les arguments que l'enseignant évoquait pour expliquer et justifier le contenu de ses communications.

\footnotetext{
${ }^{4}$ L'exclamation correspond au caractère brusque et tonique d'un énoncé verbal, exprimant de façon spontanée une émotion.

5 Le mode indicatif exprime le caractère simplement informatif d'un énoncé, sans tonalité émotionnelle particulière.
} 


\section{Résultats}

\subsection{Désignation d'entités corporelles typiques}

Pour guider les apprentissages des élèves en gymnastique, les enseignants désignent chez eux des entités corporelles particulières et récurrentes d'un enseignant à l'autre. Dix neuf termes ont été dénombrés, par lesquels ils désignent ces entités. La distribution quantitative de ces termes pour l'ensemble des huit leçons observées est représentée sur la Figure 1.

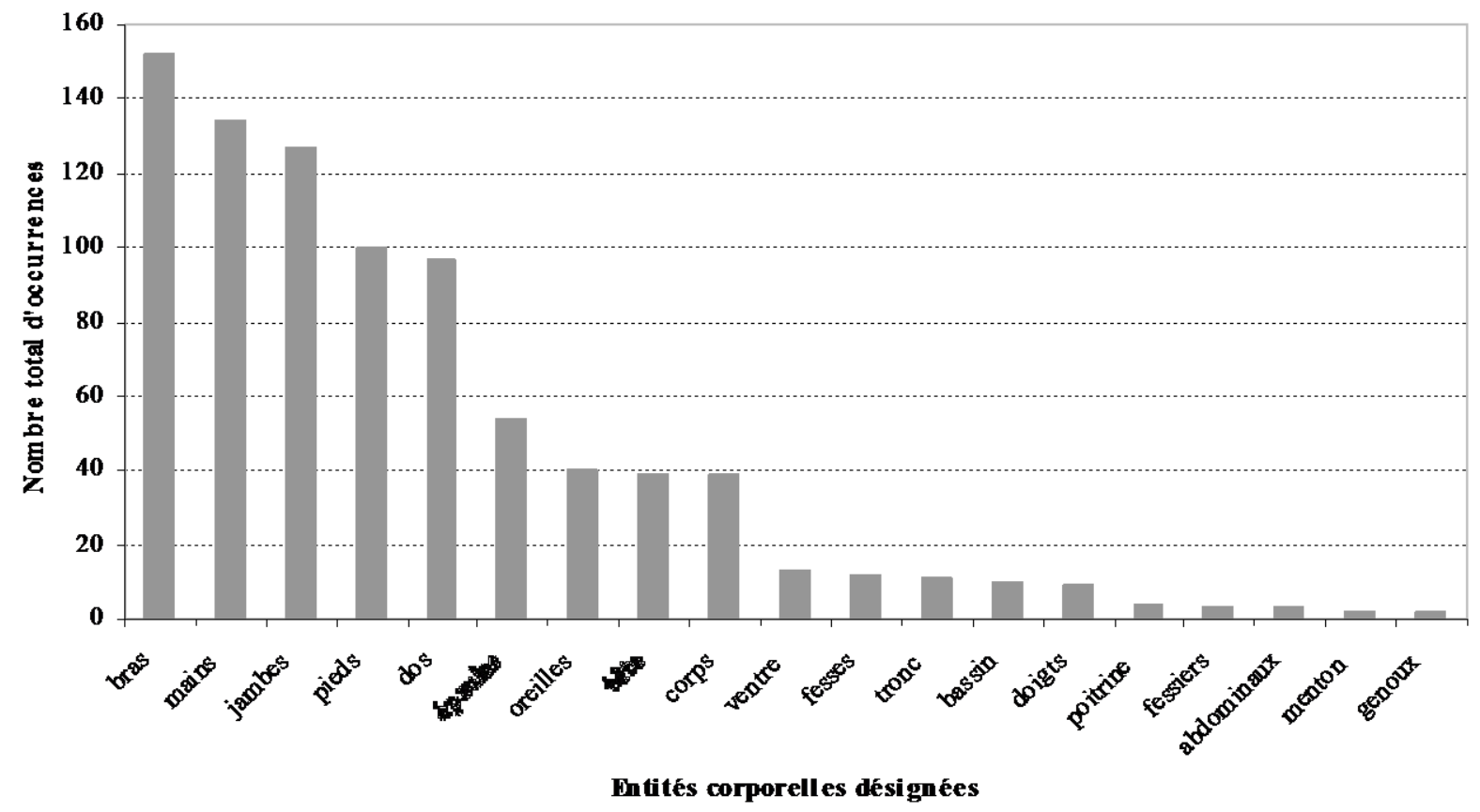

Figure 1: Distribution quantitative des entités corporelles désignées par les enseignants, au cours de 8 leçons de gymnastique

Ces résultats montrent que l'activité de guidage des apprentissages en gymnastique révèle une façon typique chez les enseignants de connaître le mouvement gymnique dans le contexte même de son enseignement : ils appréhendent le corps gymnaste selon une mosaïque de parties discrètes. La distribution quantitative de ces différents termes utilisés n'est cependant pas homogène, les entités corporelles représentant les membres étant énoncées de façon dominante. Quatre termes désignant les membres ou leurs parties constitutives (les bras, les mains, les jambes, les pieds) représentent $60 \%$ des occurrences de tous les termes employés pour désigner des parties corporelles.

Certaines parties du corps, comme les oreilles ou le menton, étaient typiquement utilisées par les enseignants comme marqueurs topographiques pour prescrire aux élèves une posture particulière à adopter. L'expression « bras aux oreilles! » était par exemple utilisée pour prescrire de façon très concise la position des bras alignée avec le tronc, tout en donnant aux élèves les indicateurs corporels précis d'identification de cette posture. D'autres expressions comme «menton à la poitrine! » ou « genoux poitrine! » avaient une fonction similaire. 


\subsection{Injonctions de formes corporelles typiques}

Tout en désignant ces différentes parties du corps, les enseignants donnaient aussi aux élèves des indications sur les mouvements à effectuer. Ces indications étaient formulées sous la forme d'injonctions, ce que montrent les résultats de l'analyse stylistique : pour l'ensemble des communications verbales des huit enseignants, le style est vif avec des phrases courtes (6 mots en moyenne par phrase) ; la proportion d'exclamatives est importante (50\% en moyenne) ; la proportion de verbes ( $37 \%$ en moyenne), très supérieure à la moyenne des styles communément reconnus ${ }^{6}$, dénote un style efficace et dynamique ; la structure grammaticale est simplifiée. Ces injonctions se résument souvent à un seul terme (allez! ; hop! ; tendu! ; pousse!), ou à des associations de mots sans construction de phrase (bras aux oreilles! ; menton poitrine! ; jambes tendues !).

Au plan locutoire, l'étude a permis de dénombrer 36 termes utilisés par les enseignants pour qualifier les mouvements des élèves, et trois catégories sont apparues adéquates pour les répertorier : i) la catégorie géométrique, dont les termes indiquent une position ou une forme du corps ; ii) la catégorie cinématique, dont les termes indiquent un déplacement du corps ; iii) la catégorie dynamique, dont les termes fournissent des indications sur la consistance du corps $^{7}$. Le Tableau 1 fournit la liste des termes par catégorie, chacun accompagné du nombre d'occurrences pour les 8 leçons observées.

\footnotetext{
${ }^{6}$ Dans l'analyse stylistique proposée par le logiciel Cordial Pro, les Styles les plus communément reconnus sont les suivants : littéraire $(27.6 \%$ de verbes en moyenne); journalistique $(28.8 \%)$; technique $(28 \%)$; juridique $(24.5 \%)$; commercial $(26.3 \%)$.

${ }^{7}$ Le terme «dynamique » nous est apparu adéquat pour signifier l'idée de consistance du corps, au sens où l'intensité des forces musculaires mises en jeu assurent plus ou moins la cohésion des différentes parties corporelles.
} 


\begin{tabular}{|c|c|c|c|}
\hline & \multicolumn{3}{|c|}{ Catégories } \\
\hline & Géométrique & Cinématique & Dynamique \\
\hline \multirow{15}{*}{$\begin{array}{l}\text { Termes utilisés pour } \\
\text { indiquer une } \\
\text { prescription (nombre } \\
\text { d'occurrences) }\end{array}$} & tendu (109) & (re)pousser (33) & dur (22) \\
\hline & serré (92) & grandir (22) & tonique (12) \\
\hline & collé (4) & tenir $(20)$ & gainé (3) \\
\hline & rentré (20) & (re)lever (16) & \\
\hline & droit (16) & (re)bondir (11) & \\
\hline & placé (11) & décoller (4) & \\
\hline & aligné (7) & poser (27) & \\
\hline & & lancer (25) & \\
\hline & & rester (24) & \\
\hline & & toucher (16) & \\
\hline & & (en)rouler (13) & \\
\hline & & avancer (7) & \\
\hline & & pencher (3) & \\
\hline & & tirer (3) & \\
\hline & & allonger (2) & \\
\hline \multirow{5}{*}{$\begin{array}{l}\text { Termes utilisés pour } \\
\text { indiquer une } \\
\text { proscription (nombre } \\
\text { d'occurrences) }\end{array}$} & fléchi (17) & tomber (23) & mou (3) \\
\hline & écarté (22) & baisser (13) & relâché (11) \\
\hline & sorti (6) & descendre (12) & \\
\hline & plié (31) & (s')écrouler (2) & \\
\hline & cambré (10) & & \\
\hline
\end{tabular}

Tableau 1 : Termes utilisés par les enseignants et fréquences de leur utilisation, au cours de 8 leçons de gymnastique, pour donner aux élèves des indications de type géométrique, cinématique et dynamique.

Au sein de chaque catégorie, le Tableau 1 montre que les termes peuvent être rangés en deux séries de significations opposées par lesquelles les enseignants contrastaient pour les élèves ce qu'ils prescrivaient ou proscrivaient. Par exemple, ils indiquaient :

«Si tu plies tes jambes tu t'écroules. Les jambes bien tendues! »;

«Reste bien tonique à l'équilibre, ne relâche pas »;

«Là tu ne les serres pas! tu te ramollis ce qui fait que ça tombe! ça tombe! ».

Les résultats montrent ainsi que les enseignants donnaient leurs instructions en gymnastique en utilisant une nomenclature ordonnée de façon binaire : plié / tendu ; tonique / relâché ; serré / ramolli ; etc. 


\subsection{Prescription de règles causales typiques}

En même temps qu'ils utilisaient le vocabulaire présenté ci-dessus, les enseignants accompagnaient souvent leurs indications en énonçant aux élèves des règles causales ${ }^{8}$. Par celles-ci, ils leur prescrivaient des principes techniques particuliers. Ces règles consistaient à mettre en relation les différents aspects géométriques, cinématiques et dynamiques des mouvements gymniques des élèves. Les résultats permettent de mettre en évidence quatre types de relations causales énoncées en classe par les enseignants à leurs élèves : relations entre différentes positions ou formes du corps ; entre formes, ou positions du corps, et déplacements corporels ; entre formes, ou positions du corps, et consistance corporelle ; entre consistance du corps et déplacements corporels. Ces quatre types de relations causales sont illustrées dans le Tableau 2.

\footnotetext{
${ }^{8}$ Le terme « règle » indique ici que, par ces énoncés, les enseignants prescrivent aux élèves ce qui doit être fait, c'est-à-dire qu'ils leur donnent une indication à suivre obligatoirement pour réaliser correctement l'action demandée.
} 


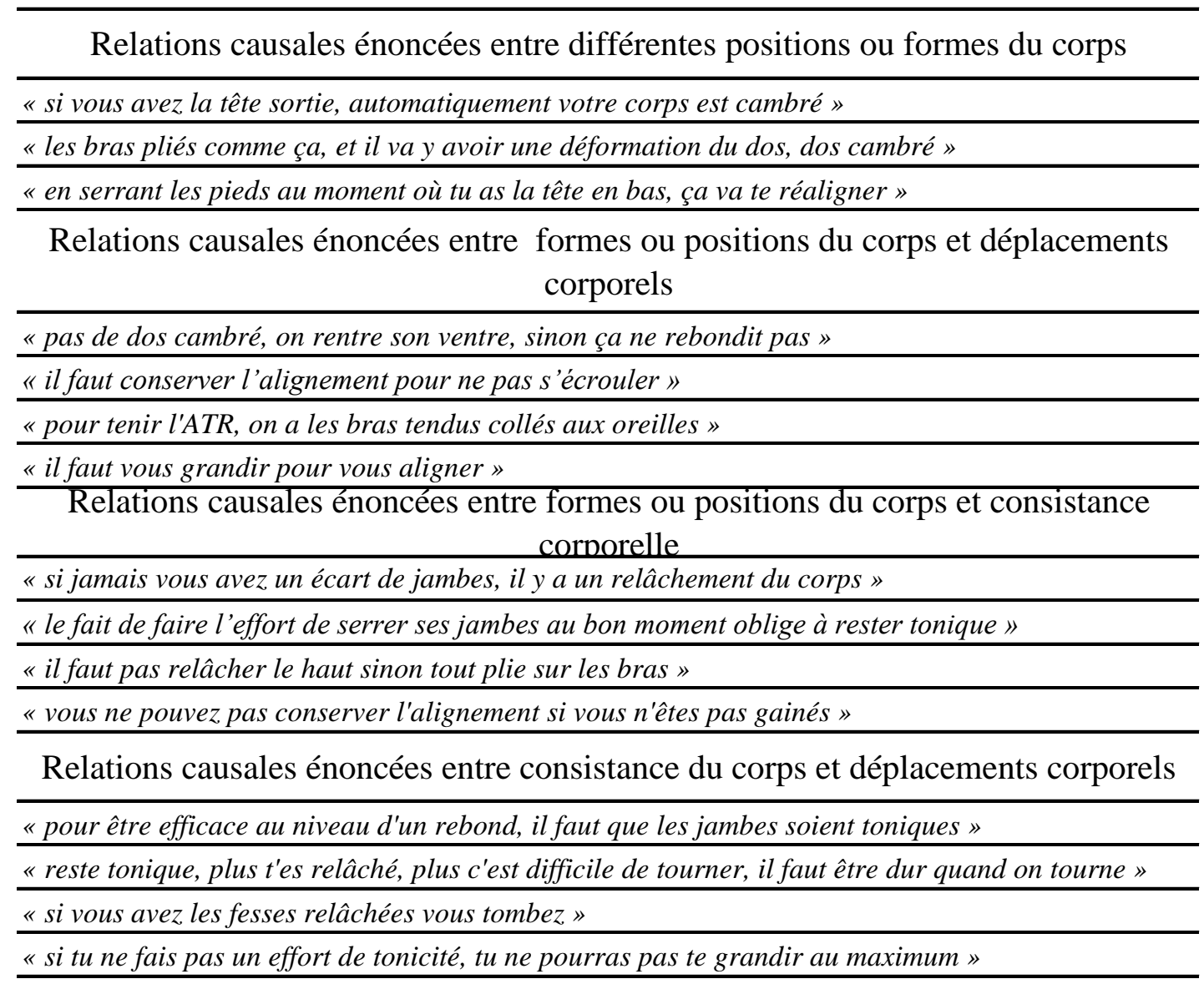

Tableau 2 : Les quatre types de relations causales énoncées par les enseignants à leurs élèves en cours de gymnastique

Les résultats ont révélé qu'en dépit de la variété des règles causales énoncées par les enseignants en classe, celles-ci reposaient sur une structure invariante : les postures fléchies, les membres écartés, les relâchements, la mollesse du corps, étaient mis en relation avec des déplacements vers le bas, des chutes ; alors que les postures tendues, les membres serrés, le gainage et la dureté du corps correspondaient à des grandissements du corps et son déplacement vers le haut. Cette constance concernant le contenu des règles exprimées peut être synthétisée selon les relations causales suivantes (Figure 2) : 


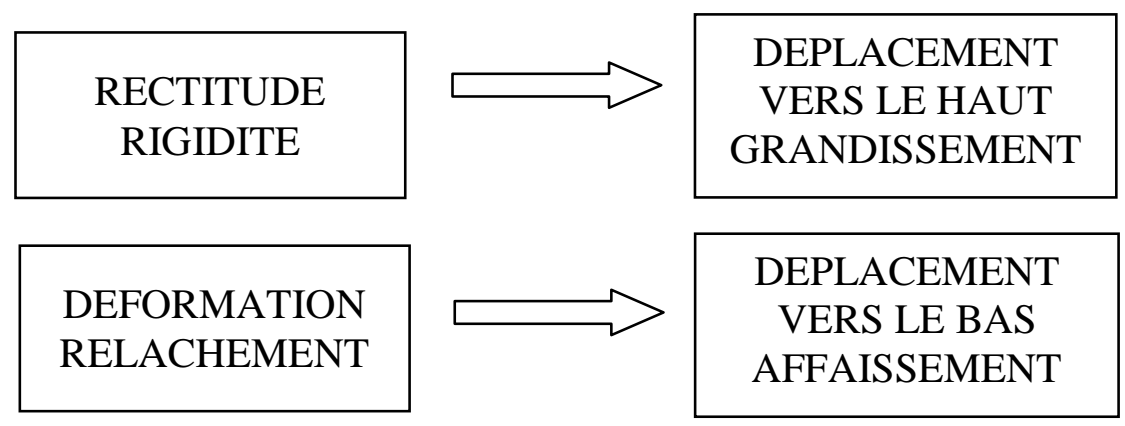

Figure 2 : Structure des règles causales énoncées par les enseignants en cours de gymnastique

Ces résultats montrent que les enseignants manifestent une connaissance portant sur des relations causales entre les propriétés «morphologiques ${ }^{9} \mathrm{du}$ corps et les déplacements corporels. Les règles causales énoncées relient globalement les déformations et les relâchements du corps avec des déplacements vers le bas, et la rectitude et la rigidité du corps avec des déplacements vers le haut.

\subsection{Argumentation des enseignants sur leurs communications en classe}

Pour commenter et expliquer le contenu de leurs communications en classe, les enseignants ont fait référence à des catégories de connaissance qu'ils ont qualifiées de mécanique, d'anatomie, et de perception :

Enseignant A : "pour pouvoir tenir en ATR, il faut être aligné, et pour ça, il faut se grandir au maximum du bout des doigts jusqu'au bout des pieds, c'est tout simplement mécanique »;

Enseignant $\mathrm{H}:$ : le cambré avec le bassin en antéversion, alors là on va se détruire le dos... au plan de l'anatomie, je pense que c'est carrément dangereux»;

Enseignant $\mathrm{D}:$ : ils ne contrôlent pas leurs jambes, c'est qu'ils ont un problème de perception ».

Invités à préciser davantage le contenu des expressions qu'ils utilisaient, les enseignants ont fait référence à deux types de connaissances : des connaissances de type théorique, et des connaissances de type métaphorique. Pour les premières, les enseignants se sont trouvés en difficulté, alors qu'ils s'engageaient eux-mêmes dans les explications théoriques, pour préciser le contenu des expressions auxquelles ils recouraient lors de l'entretien. Dans le

\footnotetext{
${ }^{9}$ Le terme « morphologique » a été choisi pour rassembler de façon synthétique les caractéristiques de forme et de consistance du corps énoncées par les enseignants.
} 
domaine mécanique par exemple, ils utilisaient la notion de transfert d'énergie, sans toujours pouvoir exploiter cette notion dans sa signification scientifique comme argument pour justifier leurs instructions en classe.

Enseignant $\mathrm{F}$ : "j'ai du mal à l'expliquer au niveau scientifique, mais je pense que ça doit être faisable quand même, je suis persuadé que si on n'est pas aligné, il y aura des fuites d'énergie, il n'y aura pas de transfert d'énergie ».

Les enseignants ont éprouvé des difficultés semblables au niveau des deux autres domaines qu'ils évoquaient :

Enseignant A : «je n'ai pas vraiment d'appui théorique en anatomie, mais par contre je suis convaincu que la position cambrée c'est dangereux pour la colonne »;

Enseignant $\mathrm{G}$ : «la question des repères, c'est clair pour moi, mais je suis pas sûr de convaincre quelqu'un de ma conception à partir du moment où je m'appuie sur un argument comme la perception qui est tout de même flou ».

Au cours de leur argumentation, les enseignants ont eu plus de facilité pour préciser le contenu des expressions qu'ils utilisaient en recourant à des métaphores et des images, ou à des concepts scientifiques mais en les utilisant dans leur signification métaphorique.

Dans le domaine mécanique, ils ont par exemple argumenté leur prescription de l'alignement du corps, en rapportant la notion de transfert d'énergie à une image, celle du mètre de charpentier, ou du bâton articulé :

Enseignant A : «quand on veut rebondir, si c'est un bâton vertical, ça va, si c'est un bâton articulé avec des angles fermés, quand le bâton tombe, il s'écrase et il ne rebondit pas... donc j'utilise un peu l'image pour expliquer ça»;

Enseignant $\mathrm{C}:$ " je prends souvent l'exemple d'un mètre de charpentier, si le mètre est bien empilé, on va appuyer dessus, il est assez solide, s'il y a un endroit où on fait une toute petite flexion, si on appuie dessus, ça va plier à cet endroit là... le corps humain c'est à peu près la même chose, si on est droit, c'est solide».

Dans le domaine de l'anatomie, les enseignants ont eu recours à la notion de pincement pour expliquer que la position cambrée de la colonne vertébrale était dangereuse pour l'intégrité du dos :

Enseignant $\mathrm{C}$ : "si le dos est cambré, il y a un risque de pincement de la colonne vertébrale, parce que la colonne se plie dans un sens et pas dans l'autre, la forme de la vertèbre fait qu'on peut ne pas fermer l'angle arrière, les lombaires elles ne sont pas prévues pour se plier en angle droit donc elles vont craquer à mon avis ».

Tel qu'il est utilisé par cet enseignant, le terme de pincement ne correspond manifestement pas à la signification qui prévaut dans le discours médical scientifique, c'est-àdire le rétrécissement d'un espace articulaire, mais plutôt à un usage métaphorique qui conçoit un pincement du disque intervertébral par ses vertèbres adjacentes. La métaphore renvoie ici à une image, celle d'une courbure du dos qui contraint, en la pinçant, sa partie concave. 
Pour étayer leur argumentation dans le domaine de la perception, les enseignants ont utilisé également de façon métaphorique le terme de repère :

Enseignant $\mathrm{E}:$ « au niveau perceptif, dès qu'on met la tête en bas, on a les repères qui ont disparu »;

Enseignant $\mathrm{D}:$ : il a des problèmes perceptifs, ça veut dire qu'il perd ses repères, ses repères visuels ou les repères sur son corps ».

Le terme de repère est utilisé ici par les enseignants comme désignant une substance que l'on peut perdre, ou qui peut disparaître dès que le corps est en rotation ou en situation renversée. La métaphore du repère leur permet ainsi d'expliciter ce qu'ils entendent par perception.

Enseignant G : " on parle de ce qui est perception, heu bon, c'est pas matériel et c'est difficile autrement que par images de pouvoir justifier ce qu'on dit, j'en suis intimement convaincu en tant que gymnaste, c'est une référence qui est intime ça, il n'y a pas tellement d'explication à donner... on peut s'en rendre compte par exemple lorsqu'on perd ses repères pour tourner vers l'avant... avant, lorsque je faisais un salto il n'y avait pas de problème, maintenant j'ai l'impression de plus trop savoir où je suis ».

En utilisant un langage métaphorique, les enseignants montrent qu'ils analysent les habiletés gymniques en se référant à des objets (le bâton, le mètre de charpentier) ou des images (image du pincement, du repère). Le dernier extrait d'entretien reporté ci-dessus montre que l'image ne renvoie pas seulement à l'idée d'une représentation imagée d'un concept, mais aussi au fait qu'il puisse être compris en référence à des sensations internes vécues. C'est typiquement le cas pour le concept de repère, que l'enseignant explicite en se référant à ses propres sensations vécues de pratiquant de gymnastique.

\section{Discussion}

Cette étude avait pour objectif d'analyser la connaissance pédagogique des contenus qu'utilisent les enseignants d'Education physique expérimentés en gymnastique pour enseigner cette matière à des élèves de collège. Elle a cherché à examiner plus précisément la connaissance des habiletés gymniques leur permettant de guider et d'aider les élèves à apprendre pendant qu'ils pratiquaient. Pour cela, elle s'est centrée sur deux points : la façon dont cette connaissance est structurée ; son lien à l'action et au contexte de la classe.

\subsection{Une connaissance de la matière organisée et intégrée}

Les résultats de cette étude révèlent que les enseignants guident l'activité d'apprentissage de leurs élèves en s'appuyant sur une connaissance des habiletés gymniques fortement structurée et contextualisée. Ils rejoignent ceux de recherches antérieures ayant montré que la connaissance des enseignants expérimentés est hautement organisée et intégrée (Housner \& Griffey, 1985 ; Rovegno et al., 2003 ; Schempp, Manross, Tan, \& Fincher, 1998 ; Tan, 1996). 
Ils contribuent aussi au développement de la recherche examinant la connaissance dans la pratique, c'est-à-dire insérée dans l'activité d'enseignement en classe.

La connaissance pédagogique de la matière chez les enseignants d'EP expérimentés peut être caractérisée comme une connaissance morpho-logique ${ }^{10}$ de la gymnastique, structurée par des formes typiques du corps et des mouvements des élèves, elles-mêmes organisées de façon binaire à partir des catégories de rectitude / flexion, rigidité / relâchement. Elle reflète des relations entre des formes corporelles typiques et l'efficacité des mouvements gymniques qu'elles induisent : un corps ou des segments droits et rigides génèrent un déplacement aérien et efficace du gymnaste ; un corps ou des segments fléchis et mous provoquent un écrasement ou un déplacement inefficace vers le bas. Les enseignants rapportent ainsi la complexité de l'analyse des mouvements gymniques à un système de corrélations empiriques portant sur des «types formels », c'est-à-dire des configurations ${ }^{11}$ typiques des mouvements gymniques (Dubois, Fleury, \& Mazet, 1993 ; Gal-Petitfaux \& Durand, 2001 ; Mazet, 1991). La connaissance qu'ont les enseignants des habiletés gymniques qu'ils enseignent est structurée par des «totalités signifiantes » (Barbier, 2000, p. 96), ou plus précisément des « formes signifiantes » (Zeitler, 2003) typiques, qui leur permettent de se rendre intelligibles les actions gymniques produites par les élèves en classe.

En termes phénoménologiques, ces configurations fondent la relevance interprétative (Schütz, 1987) des enseignants lorsqu'ils sont en situation de guidage des apprentissages des élèves, c'est-à-dire ce par quoi ils peuvent donner un sens aux mouvements corporels qu'ils observent. Cette connaissance qu'ont les enseignants experts de la matière leur permet de reconnaître rapidement, en contexte, les aspects pertinents des conduites gymniques des élèves et les interpréter en termes d'efficacité. Les résultats confirment que les PCK correspondent à l'intégration de différentes connaissances (Cochran et al., 1993) ; notamment dans cette étude les enseignants experts ont intégré une connaissance de la matière, la gymnastique, avec une connaissance spécifique de l'activité des élèves, en particulier des formes corporelles gymniques qu'ils produisent en classe.

\subsection{Une connaissance fondée sur la reconnaissance perceptive}

Les résultats montrent que les enseignants expérimentés en gymnastique interprètent immédiatement les mouvements gymniques qu'ils perçoivent en classe. Autrement dit, ils perçoivent et reconnaissent directement les formes de corps que les élèves donnent à voir comme des significations $^{12}$. Ce ne sont donc pas seulement des attributs physiques qu'ils perçoivent, mais plus directement des significations. Ce résultat est conforme à ceux des recherches sur les caractéristiques des enseignants experts. Ces derniers reconnaissent et interprètent les événements se déroulant en classe avec une grande rapidité et précision

\footnotetext{
${ }^{10}$ La séparation des deux termes morpho et logique par un tiret souligne qu'il s'agit d'une connaissance portant sur la logique de la forme.

${ }^{11}$ Le terme «configuration » doit être pris ici au sens d'une «organisation singulière de formes régulières » (Barbier, 2000, p. 100).

${ }^{12}$ Il convient de distinguer ici entre perception directe et perception immédiate de significations (Quéré, 1999). Dire que la perception de signification est directe signifie qu'elle n'advient pas après coup, en ajoutant au matériau sensoriel une interprétation. Elle peut être directe mais non immédiate, en étant notamment médiatisée par des connaissances.
} 
(Carter, Cushing, Sabers, Stein, \& Berliner, 1988 ; Leinhardt \& Greeno, 1986). Ils ont développé des habiletés de discrimination fine, spécifique à des domaines particuliers de la matière, leur permettant de reconnaître rapidement des événements pertinents en classe et d'y réagir rapidement. Une aptitude de l'expert nommée « reconnaissance de similarité globale » (Dreyfus \& Dreyfus, 1988) lui permet de reconnaître immédiatement la « signature » d'un évènement, de l'interpréter et d'agir en conséquence (Tochon, 1993, p. 130). L'expert « voit directement ce qui est pertinent dans la situation parce que celle-ci rappelle un modèle d'expérience passée, déjà formé en termes de conséquences » (p. 130). L'expérience joue un rôle essentiel dans l'acquisition de ces connaissances en développant « l'aptitude de reconnaître des patrons de situations et d'avoir la réaction adéquate sans recours à l'analyse » (p. 157).

Cette connaissance directe, ne reposant pas sur une décomposition analytique et demandant très peu d'inférence cognitive caractérise l'arationalité de l'expert (Tochon, 1993). Il s'agirait d'une inférence abductive, faite de jugements plus perceptuels que cognitifs, antérieurs à la pensée critique (Tochon, 1993). Il pourrait s'agir d'une perception protocognitive, chaînon intermédiaire entre deux façons de recevoir l'information : la manière intensive de la perception sensorielle, et la manière discursive de la perception cognitive (Proust, 1997). Dans le prolongement de la psychologie de la forme, la question de la perception directe d'une signification a été discutée à partir des propositions théoriques de Wittgenstein (1994). En référence à la façon dont on perçoit directement l'expression d'un visage, la notion de «perception physionomique » est utilisée pour désigner les cas où l'interprétation d'une signification est immédiate, c'est-à-dire qui ne passe pas par le détour d'une inférence. La perception physionomique d'une forme est un « voir comme... » (Wittgenstein, 1994). L'interprétation y est absorbée de telle sorte qu'elle apparaît encore comme une perception et non comme un acte mental qui en serait séparé (Bouveresse, 1995).

Les enseignants spécialistes de gymnastique voient les formes de corps comme des significations, c'est-à-dire les interprètent directement en termes d'efficacité des habiletés gymniques. Les résultats ont montré que pour argumenter leurs communications en classe, ils ont fait appel à des connaissances métaphoriques. Le recours à ces connaissances manifeste un raisonnement analogique, en mettant en relation un « voir comme... » métaphorique, avec un « voir comme... » vécu en situation d'instruction. Pour justifier et rendre compte de la connaissance qui structurait leurs instructions en classe, les enseignants ont utilisé des concepts (énergie, mécanique, transfert d'énergie, perception, etc.) qu'ils ont néanmoins rapportés à des métaphores de substance (Lakoff \& Johnson, 1985 ; Munby, 1986) ou des images (Clandinin, 1986 ; Johnston, 1992) : les métaphores du repère comme substance que l'on perd ou construit ou de l'énergie comme substance qui fuit; les images du bâton articulé ou du mètre de charpentier. Les métaphores et les images facilitent le lien que les enseignants font entre leurs explications et justifications de type théorique, avec leur activité d'instruction en classe. En les rendant substantiels par la métaphore ou l'image, les enseignants rapportent les concepts théoriques à des entités matérielles, tout comme sont matériels les attributs physiques des mouvements gymniques qu'ils perçoivent en classe. Leur raisonnement analogique est ainsi facilité par la congruence de la nature matérielle des entités qu'ils mettent en relation. Le trait d'union entre la connaissance mobilisée a posteriori et celle présente dans l'action en classe est le « voir comme »: «voir comme » du raisonnement métaphorique et « voir comme » de la perception physionomique (Bouveresse, 1995). 


\subsection{Une connaissance structurée par et pour l'action}

Les formes de corps perçues par les enseignants expérimentés en gymnastique sont des affordances (Gibson, 1979). Celles-ci ont une valeur fonctionnelle, au sens où elles sont des points d'appui permettant aux enseignants d'intervenir pour guider les apprentissages des élèves. Percevoir les formes des corps et les mouvements gymniques comme des significations revient à saisir des potentialités d'action d'intervention dans l'environnement de la classe. La façon dont les enseignants connaissent la gymnastique a une valeur fonctionnelle en classe. Elle leur sert à attribuer du sens aux actions gymniques des élèves au moment même où ceux-ci les réalisent, et à savoir comment intervenir. Autrement dit, les formes signifiantes perçues ont un caractère fonctionnel qui se traduit «par une fonction d'orientation de l'action par la forme » (Zeitler, 2003). La reconnaissance perceptive des formes des mouvements gymniques comme signification permet de comprendre comment s'opère le couplage entre la connaissance, l'action et le contexte de classe : la forme perçue, re-connue comme typique, dispose l'enseignant à agir d'une certaine façon

(Dubois et al., 1993 ; Mazet, 1991). Ce couplage connaissance - action, reposant sur la perception directe de la signification des actions des élèves, caractérise un « sens de la typicalité » (Tochon, 1993) : c'est par celui-ci que les enseignants experts appréhendent directement les propriétés fonctionnelles des situations de classe.

Les résultats montrent que la façon dont les enseignants communiquent avec leurs élèves pour les instruire repose largement sur des injonctions, en particulier des prescriptions de formes du corps. Pour enseigner les habiletés gymniques, ils font agir les élèves en les incitant à produire les formes qu'ils attendent et reconnaissent. En utilisant un vocabulaire usuel, et en formulant des prescriptions de forme nettement contrastées (e.g., Serrez les jambes !; Ne pliez pas, sinon ça s'écroule !; Bras aux oreilles ! Corps bien dur si tu veux tourner !), les enseignants s'expriment clairement, simplement, et de façon concise auprès des élèves.

Ces communications sous forme d'injonctions sont des actes de langage qui conjuguent des aspects locutoires, et des aspects illocutoires et perlocutoires (Austin, 1970 ; Searle, 1996). La forte proportion de phrases exclamatives montre que les enseignants activent les élèves en leur parlant, au sens où ils suscitent chez eux des engagements (Gal-Petitfaux \& Saury, 2002) à réaliser l'habileté gymnique et pas seulement à entendre l'instruction donnée pour l'exécuter. La fonction des injonctions est ici à la fois référentielle (l'enseignant indique par une locution ce qu'il faut faire), illocutoire (par un acte de langage directif, il signifie à l'élève qu'il veut obtenir une réponse) et perlocutoire (il incite l'élève à faire l'action motrice). Instruire les élèves revient ici à les faire agir de façon conforme (au sens strict du terme). La présence massive de phrases exclamatives dans les communications des enseignants en classe aide à mieux comprendre la relation des PCK en gymnastique à l'activité de l'enseignant lorsqu'il guide les apprentissages techniques des élèves. Ces PCK ont une structure particulière qui peut s'expliquer par deux raisons : d'une part, elles ne sont pas isolables du contexte pratique de leur usage, c'est-à-dire de la situation de classe ; d'autre part, la matière qui est enseignée en EP ne consiste pas seulement à faire comprendre aux élèves les aspects relatifs aux activités sportives supports, mais à les inciter à agir et à réaliser efficacement les habiletés motrices (Siedentop, 2002).

\section{Conclusion}


Les résultats de cette étude ont montré que pour communiquer oralement leurs instructions, les enseignants d'EPS expérimentés en gymnastique se référaient à une connaissance morpho-logique des habiletés gymniques. Cette connaissance organise la perception qu'ils ont des formes de corps et de mouvement que produisent les élèves en classe, en leur permettant de les interpréter directement comme formes signifiantes typiques. Le rôle central joué par la perception des formes dans le couplage entre la connaissance et les interventions des enseignants en classe permet de comprendre l'importance, soulignée par les chercheurs, du caractère situé de cette connaissance. Il serait intéressant de mener des recherches complémentaires pour voir si des processus comparables de reconnaissance de forme sont repérables dans l'enseignement d'autres activités sportives.

Cette étude permet aussi de mieux comprendre l'importance de l'expérience professionnelle dans l'intégration des PCK PE à la pratique. La difficulté des enseignants débutants pour intégrer des PCK PE à leur pratique est liée en partie à leur manque de disponibilité, en classe, pour observer les comportements des élèves. Toutefois, lorsqu'elle est rendue possible, il leur reste à savoir quoi regarder et comment l'interpréter. L'étude des connaissances des enseignants expérimentés en gymnastique montre que l'habileté perceptive qu'ils ont construite pour observer et instruire les élèves relève d'une reconnaissance de formes. Plutôt que de juger a priori comme superficielle cette façon d'appréhender les habiletés motrices (Marsenach, 1991), il serait fécond pour la formation des enseignants de s'intéresser à la manière de les entraîner à identifier, typifier et interpréter en situation les formes gymniques que donnent à voir les élèves. Cette façon de connaître la matière pour l'enseigner ne préjuge pas de la démarche pédagogique de l'enseignant, ni des objectifs qu'il poursuit pour faire l'Education Physique des élèves. En particulier, le fait que la connaissance de la matière soit structurée par des typifications de formes ne veut pas dire que les enseignants transmettent obligatoirement telles quelles, en tant que contenus, des formes de mouvements gymniques repérées par exemple chez les gymnastes de haut niveau. En d'autres termes, le fait que les enseignants guident les élèves pour leur faire apprendre les habiletés gymniques en les faisant agir selon des prescriptions de forme ne veut pas dire qu'ils leur enseignent à assimiler ces habiletés comme des formes à reproduire, c'est-à-dire qu'ils adoptent une démarche d'enseignement formelle en gymnastique (Marsenach, 1991 ; Perez, 1998). 


\section{Références}

Amade-Escot, C. (1998). Les contenus de l'éducation physique, contribution de deux programmes de recherche : "Pedagogical Content Knowledge" et "didactique de l'Education Physique”. Science et Motricité, 32-33, 16-26.

Austin, J.L. (1970). Quand dire, c'est faire. Paris: Seuil.

Barbier, J.M. (2000). Sémantique de l'action et sémantique d'intelligibilité des actions. In B. Maggi (Dir.). Manières de penser, manières d'agir en éducation et en formation (pp. 89104). Paris: PUF.

Barrett, K.R., \& Collie, S. (1996). Children learning lacrosse from teachers learning to teach it : The discovery of pedagogical content knowledge by observing children's movement. Research Quarterly for Exercise and Sport, 67(3), 297-309.

Baxter, J.A., \& Lederman, N.G. (1999). Assessment and measurement of pedagogical content knowledge. In J. Gess-Newsome \& N.G. Lederman (Eds.), Examining pedagogical content knowledge (pp. 147-162). Dordrecht: Kluwer Academic Publishers.

Bouveresse, J. (1995). Langage, perception et réalité. Tome 1. La perception et le jugement. Nîmes: J. Chambon Editions.

Carnus, M.F. (2002). Croyances, conceptions, intentions et pratiques usuelles dans l'enseignement de la gymnastique : le cas de l'ATR et du repérage. In J.F. Robin \& A. Durny (Dir.), Travaux d'actualité en activités gymniques et acrobatiques (pp. 160-164). Dossier EPS, 57. Paris : Revue EPS.

Carter, K., Cushing, K., Sabers, D., Stein, P., \& Berliner, D. (1988). Expert-novice differences in perceiving and processing visual classroom information. Journal of Teacher Education, 39(3), 25-31.

Cizeron, M., \& Gal-Petitfaux, N. (2002). Croyances factuelles et croyances représentationnelles en enseignement scolaire de la gymnastique. STAPS, 59(23), 43-56.

Cizeron, M., \& Gal-Petitfaux, N. (2003). Savoirs d'action et savoirs de justification en situation d'enseignement : le cas de la gymnastique. Revue Française de Pédagogie, 143, 91100.

Clandinin, D.J. (1986). Classroom practice: teacher images in action. Philadelphia: Falmer Press.

Cochran, K.F., De Ruiter, J.A., \& King, R.A. (1993). Pedagogical content knowing: an integrative model for teacher preparation. Journal of Teacher Education, 44(4), 263-272.

Coulon, A. (1988). Ethnométhodologie et enseignement. Revue Française de Pédagogie, 82, 65-101. 
Dodds, P. (1995). Pedagogical content knowledge as the central problem of teacher education. Paper adressed to Teacher Education Conference. Morgatown, Nov. 26-29, USA.

Dreyfus, S.E., \& Dreyfus, H.L. (1988). Towards a reconciliation of phenomenology and AI. In D. Partridge \& Y.A. Wilks (Eds.). Foundational issues in artificial intelligence (pp. 471-499). Cambridge: University Press.

Dubois, D., Fleury, D., \& Mazet, C. (1993). Représentations catégorielles : perception et/ou action ? In A. Weill-Fassina, P. Rabardel, \& D. Dubois (Eds.), Représentations pour l'action (pp. 79-93). Toulouse: Octarès.

Durand, M. (1996). L'enseignement en milieu scolaire. Paris: PUF.

Gal-Petitfaux, N., \& Durand, M. (2001). L'enseignement de l'Education Physique comme "action située": propositions pour une approche d'anthropologie cognitive, STAPS, 55(22), 79-100.

Gal-Petitfaux, N., \& Saury, J. (2002). L'agir professionnel en éducation physique et en sport dans une perspective d'anthropologie cognitive. Revue Française de Pédagogie, 138, 51-61.

Gauthier, C., Desbiens, J.F., Malo, A., Martineau, S., \& Simard, D. (1997). Pour une théorie de la pédagogie. Recherches contemporaines sur le savoir des enseignants. Québec: Les Presses de l'Université de Laval.

Gess-Newsome, J. (1999). Pedagogical content knowledge: An introduction and orientation. In J. Gess-Newsome \& N.G. Lederman (Eds.), Examining pedagogical content knowledge (pp. 3-20). Dordrecht: Kluwer Academic Publishers.

Gess-Newsome, J., \& Lederman, N.G. (1999). Examining pedagogical content knowledge. Dordrecht: Kluwer Academic Publishers.

Gibson, J.J. (1979). The ecological approach to visual perception. Boston: Houghton Mifflin.

Graber, K.C. (1995). The influence of teacher education programs on the beliefs of student teachers: General pedagogical knowledge, pedagogical content knowledge, and teacher education course work. Journal of Teaching in Physical Education, 14(2), 157-178.

Graber, K.C. (2001). Research on teaching in physical education. In V. Richardson (Eds.). Handbook of research on teaching (pp. 491-519). Washington: AERA.

Grosjean, M., \& Lacoste, M. (1999). Communication et intelligence collective. Le travail à l'hôpital. Paris: PUF.

Grossman, P.L. (1990). The making of a teacher: Teacher knowledge and teacher education. New-York: Teacher College Press.

Gudmundsdottir, S. (1990). Values in pedagogical content knowledge. Journal of Teacher Education, 41(3), 44-52. 
Housner, L.D., \& Griffey, D.C. (1985). Teacher cognition: Differences in planning and interactive decision making between experienced and inexperienced teachers. Research Quarterly for Exercice and Sport, 56(1), 45-53.

Jenkins, J.M., \& Lou Veal, M. (2002). Preservice teacher's PCK development during peer coaching. Journal of Teaching in Physical Education, 22(1), 49-68.

Johnston, S. (1992). Images: a way of understanding the practical knowledge of student teachers. Teaching and Teacher Education, 8(2), 123-136.

Kulinna, P.H., Silverman, S., \& Keating, X.D. (2000). Relationship between teachers' belief systems and actions toward teaching physical activity and fitness. Journal of Teaching in Physical Education, 19(2), 206-221.

L'Ecuyer, R. (1988). L'analyse de contenu : notion et étapes. In J.P. Delauriers (Dir.), Les méthodes de la recherche qualitative (pp. 49-65). Québec: Presses de l'Université du Québec.

Lakoff, G., \& Johnson, M. (1985). Les métaphores dans la vie quotidienne. Paris: Editions de Minuit.

Leinhardt, G., \& Greeno, J.G. (1986). The cognitive skill of teaching. Journal of Educational Psychology, 78(2), 75-95.

Léziart, Y. (1997). Savoir savant et transposition didactique en éducation physique et sportive. STAPS, 42(18), 59-72.

Malet, R. (2000). Savoir incarné, savoir narratif. Recherche phénoménologique et formation de l'enseignant-sujet. Revue Française de Pédagogie, 132, 43-53.

Marks, R. (1990). Pedagogical content knowledge: From a mathematical case to a modified conception. Journal of Teacher Education, 42(3), 3-11.

Marsenach, J. (1991). EPS, quel enseignement? Paris: INRP.

Mazet, C. (1991). Fonctionnalité dans l'organisation catégorielle. In D. Dubois (Eds.), Sémantique et cognition. Catégories, prototypes, typicalité (pp. 89-100). Paris: CNRSEditions.

Mehan, H., \& Wood, H. (1975). The reality of ethnomethodology. New-York: Wiley.

Munby, H. (1986). Metaphor in the thinking of teachers: An exploratory study. Journal of Curriculum Studies, 18(2), 197-209.

Nouillot, P., \& Nata, F. (2002). Modèle d'organisation segmentaire et indice sur les contenus d'enseignement. Exemple de l'ATR. In J.F. Robin \& A. Durny (Dir.), Travaux d'actualité en activités gymniques et acrobatiques (pp. 102-107). Dossier EPS, 57. Paris : Revue EPS.

Perez, S. (1998). Etude du cours d'action de professeurs d'EPS expérimentés spécialistes et non spécialistes de gymnastique face à une classe de 24, 12, 5 élèves : contribution à une 
approche ergonomique de l'enseignement. Thèse non publiée de doctorat en STAPS. Montpellier: Université Montpellier I.

Proust, J. (1997). Comment l'esprit vient aux bêtes. Paris: Gallimard.

Quéré, L. (1999). Action située et perception du sens. In M. de Fornel \& L. Quéré (Dir.), La logique des situations (pp. 301-338). Raisons pratiques, 10. Paris : EHESS.

Rovegno, I. (1992). Learning in a field-based methods course: the development of pedagogical content knowledge. Teaching and Teacher Education, 8(1), 69-82.

Rovegno, I. (1994). Teaching within a curricular zone of safety: School culture and the situated nature of student teachers' pedagogical content knowledge. Research Quarterly for Exercise and Sport, 65(3), 269-279.

Robin, J.F. (1998). Les savoirs de leaders de théories didactiques pour la gymnastique scolaire. Spécificité, structure et sens des savoirs à enseigner pour la gymnastique scolaire : quatre études de cas. In J.F. Robin \& D. Hauw (Dir.), Actualités de la recherche en activités gymniques et acrobatiques (pp. 90-93). Dossier EPS, 39. Paris : Revue EPS.

Rovegno, I., Chen, W., \& Todorovich, J. (2003). Accomplished teachers' pedagogical content knowledge of teaching dribbling to third grade children. Journal of Teaching in Physical Education, 22(4), 426-449.

Schempp, P.G., Manross, D., Tan, S.K.S., \& Fincher, M.D. (1998). Subject expertise and teachers' knowledge. Journal of Teaching in Physical Education, 17(3), 342-356.

Schütz, A. (1987). Le chercheur et le quotidien. Paris : Méridiens Klincksieck.

Searle, J.R. (1996). Les actes de langage. Paris: Hermann.

Sebren, A. (1995). Preservice teachers' reflections and knowledge development in a fieldbased elementary physical education methods course. Journal of Teaching in Physical Education, 14(3), 262-283.

Shulman, L.S. (1986). Those who understand: knowledge growth in teaching. Educational Researcher, 15(2), 4-14.

Shulman, L.S. (1987). Knowledge and teaching: foundation for a new reform. Harvard Educational Review, 51(1), 1-22.

Siedentop, D. (2002). Content knowledge for Physical Education. Journal of Teaching in Physical Education, 21(4), 368-377.

Siedentop, D., \& Eldar, E. (1989). Expertise, experience and effectiveness. Journal of Teaching in Physical Education, 8(3), 254-260.

Tamir, P. (1988). Subject matter and related pedagogical knowledge in teacher education. Teaching and Teacher Education, 4(2), 99-110. 
Tan, S.K.S. (1996). Differences between experienced and inexperienced physical education teachers' augmented feedback and interactive teaching decisions. Journal of Teaching in Physical Education, 15(2), 151-170.

Tardif, M., \& Lessard, C. (1999). Le travail enseignant au quotidien. Bruxelles: De Boeck.

Tochon, F.V. (1991). L'enseignement stratégique : transformation de la connaissance dans la pensée des enseignants. Toulouse: Editions Universitaires du Sud.

Tochon, F.V. (1993). L'enseignant expert. Paris: Nathan.

Tochon, F.V. (1996). Rappel stimulé, objectivation clinique, réflexion partagée.

Fondements méthodologiques et applications pratiques de la rétroaction vidéo en recherche et en formation. Revue des Sciences de l'éducation, 22(3), 467-502.

Varela, F.J. (1989). Autonomie et connaissance. Paris : Seuil.

Wittgenstein, L. (1994). Remarques sur la philosophie de la psychologie. Vol. 2. Mauvezin: Editions T.E.R.

Zeitler, A. (2003) Emergence de types et construction de forme signifiante pour l'action chez un enseignant débutant. In J.M. Barbier \& M. Durand (Eds.), L'analyse de l'activité: approches situées. Recherche et Formation, 42, 51-62. 